\title{
Article \\ A Novel Adaptive Battery-Aware Algorithm for Data Transmission in IoT-Based Healthcare Applications
}

\author{
Hina Magsi ${ }^{1}$ (D) Ali Hassan Sodhro ${ }^{1,2,3}{ }^{\mathbb{D}}$, Mabrook S. Al-Rakhami ${ }^{4, *}$, Noman Zahid ${ }^{1}$, Sandeep Pirbhulal ${ }^{5, *}$ \\ and Lei Wang ${ }^{3}$ (D)
}

check for

updates

Citation: Magsi, H.; Sodhro, A.H.; Al-Rakhami, M.S.; Zahid, N.; Pirbhulal, S.; Wang, L. A Novel Adaptive Battery-Aware Algorithm for Data Transmission in IoT-Based Healthcare Applications. Electronics 2021, 10, 367. https://doi.org/ 10.3390/electronics10040367

Received: 28 December 2020

Accepted: 19 January 2021

Published: 3 February 2021

Publisher's Note: MDPI stays neutral with regard to jurisdictional claims in published maps and institutional affiliations.

Copyright: (c) 2021 by the authors. Licensee MDPI, Basel, Switzerland. This article is an open access article distributed under the terms and conditions of the Creative Commons Attribution (CC BY) license (https:// creativecommons.org/licenses/by/ $4.0 /)$.
1 Department of Electrical Engineering, Sukkur IBA University, Sukkur 65200, Pakistan; hina.mece17@iba-suk.edu.pk (H.M.); ali.hassan.sodhro@ltu.se (A.H.S.); noman.mece17@iba-suk.edu.pk (N.Z.)

2 Department of Computer and System Science, Mid-Sweden University, 83125 Östersund, Sweden

3 Shenzhen Institutes of Advanced Technology, Chinese Academy of Sciences, Shenzhen 518000, China; wang.lei@siat.ac.cn

4 Research Chair of Pervasive and Mobile Computing, Information Systems Department, College of Computer and Information Sciences, King Saud University, Riyadh 11543, Saudi Arabia

5 Department of Informatics, University of Beira Interior, 6201-001 Covilhã, Portugal

* Correspondence: malrakhami@ksu.edu.sa (M.S.A.-R.); sandeep.hemnani28@gmail.com (S.P.); Tel.: +966-14699506 (M.S.A.-R.)

\begin{abstract}
The internet of things (IoT) comprises various sensor nodes for monitoring physiological signals, for instance, electrocardiogram (ECG), electroencephalogram (EEG), blood pressure, and temperature, etc., with various emerging technologies such as Wi-Fi, Bluetooth and cellular networks. The IoT for medical healthcare applications forms the internet of medical things (IoMT), which comprises multiple resource-restricted wearable devices for health monitoring due to heterogeneous technological trends. The main challenge for IoMT is the energy drain and battery charge consumption in the tiny sensor devices. The non-linear behavior of the battery uses less charge; additionally, an idle time is introduced for optimizing the charge and battery lifetime, and hence the efficient recovery mechanism. The contribution of this paper is three-fold. First, a novel adaptive battery-aware algorithm (ABA) is proposed, which utilizes the charges up to its maximum limit and recovers those charges that remain unused. The proposed ABA adopts this recovery effect for enhancing energy efficiency, battery lifetime and throughput. Secondly, we propose a novel framework for IoMT based pervasive healthcare. Thirdly, we test and implement the proposed ABA and framework in a hardware platform for energy efficiency and longer battery lifetime in the IoMT. Furthermore, the transition of states is modeled by the deterministic mealy finite state machine. The Convex optimization tool in MATLAB is adopted and the proposed ABA is compared with other conventional methods such as battery recovery lifetime enhancement (BRLE). Finally, the proposed ABA enhances the energy efficiency, battery lifetime, and reliability for intelligent pervasive healthcare.
\end{abstract}

Keywords: IoMT; data transmission; intelligent healthcare; proposed ABA; BRLE

\section{Introduction}

In the modern world, a better healthcare system is the main challenge for a growing world population. The internet of medical things (IoMT) is the vision of providing a better and more pervasive healthcare system. The IoMT is the integration of medical devices through Wi-Fi and permits device-to-device (D2D) communication. In recent days, the most challenging issue is the time needed for web services. Three-dimensional (3D) video can be downloaded at sporadic intervals by keeping in mind the latest technological trends. The collected voluminous data with less delay is obtained for the accurate data measurement. It will increase the device resource allocation ability and offers quicker speed 
for the heterogenous networks. The IoMT comprises various heterogeneous networks, for instance, $\mathrm{Wi}-\mathrm{Fi}$, Bluetooth, ZigBee, and other cellular platforms. The D2D communication is the central part of the IoMT platform with high efficiency and reliability. The main traits of an intelligent healthcare system are to offer less delay and high throughput and reliability, which are very important for an effective and accurate diagnosis and consultation. The critical time analysis is the key parameter to be considered for emergency healthcare applications. The highly reliable and delay-tolerant communication and data transmission can be achieved through IoT-driven wearable devices, as shown in Figure 1. The key emerging technologies such as, sensors, IoT, and cyber physical system (CPS) are the key enablers for forming the IoMT, which is the main component for intelligent, smart, and pervasive healthcare, as shown in Figure 1.

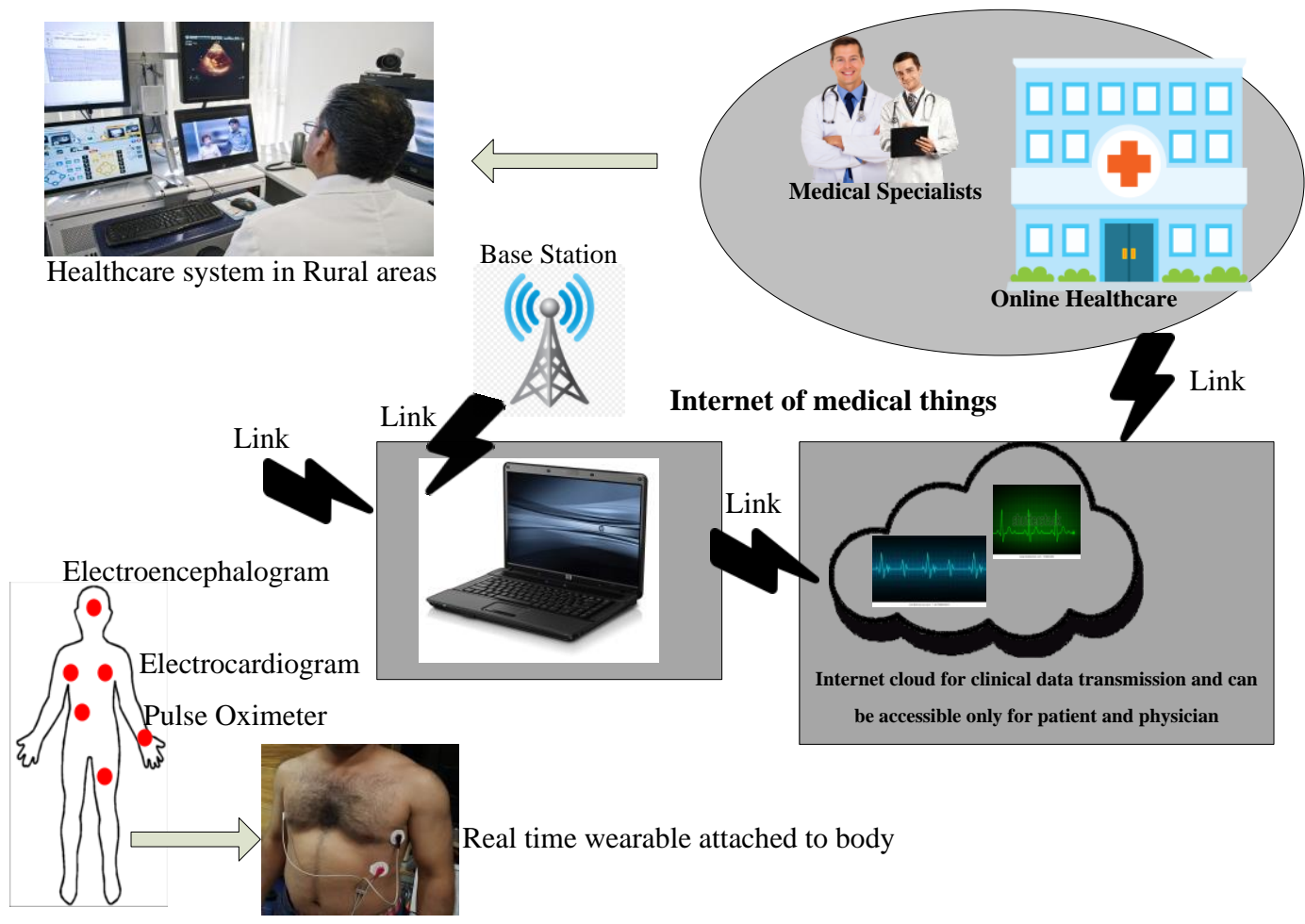

Figure 1. Proposed healthcare framework, physiological signals, images and video streams are transmitted through wearable devices from the transmitter node to the end-users, i.e., the base station over the wireless link.

The main traits of the intelligent technological trends are to fairly allocate the resources, for instance, high throughput, less delay, and high reliability. The key emerging technologies, for instance, sensors, IoT, and cyber physical systems (CPS) are key enablers to form the IoMT, which is the main component for today's intelligent pervasive medical world. The intelligent and fast networks provide high data rates and low latency which are essential for remote emergency applications. Due to the high compatibility and integrating nature of the heterogenous intelligent healthcare technologies it is necessary to have better interoperability platforms. IoMT driven intelligent healthcare is one of the examples, which has a built-in global positioning system (GPS) for adequately managing and monitoring the data traffic through portable devices, because massive data contents are collected, clustered, and examined through high-capacity sensor-based devices [1]. The human vital sign signals collection and monitoring are essential and necessary for detecting the patient's disease and then to recommend the proper cure. It is easy to collect, transmit and examine the patient's data through the sensor-based wearable devices. Still, the main challenges faced by these devices and conventional methods are not potential candidates to deal with high energy and battery charge drain, and hence shorter battery lifetime problems. Thus, it is highly important to develop the energy and battery charge optimization techniques for 
enhancing the lifetime in IoMTs to facilitate the patients and medical staff at reasonable and cost-effective rates $[2,3]$. There are various emerging features of the IoMT based intelligent pervasive healthcare platforms such as, high reliability, less delay, more visibility, better Quality of Service (QoS), and user's perception, i.e., (Quality of Experience (QoE), high flexibility and scalability. In addition, IoMT's key components are sensors, actuators, base stations (BS) and cluster nodes for big data collection and analytics through the internet.

After a rigorous review, some crucial challenges in the IoMT are found, which degrade the performance of the entire healthcare platform. These issues can be resolved to improve the performance for achieving high reliability and better facilities in intelligent healthcare. Several researchers have contributed in the IoMT, and bridge the gap between doctors, patients, and healthcare entities. In this way ease, accuracy and flexibility can be achieved, and in addition IoMT provides services to doctors and patients to work more efficiently with less effort and high intelligence.

The IoMT is one of the emerging technologies to revolutionize healthcare and that provides a platform to physicians for analyzing the critical patient's health status over a stable wireless link, because if there is a stronger link there will be more content delivery, and hence higher charge and energy drain. To understand the battery discharging process, it is vital to examine it, and the discharging process starts when the load is connected and continues till all the stored charges attached to the electrodes are utilized. The remaining charges are diffused in the battery surroundings and become unused. If some idle time is inserted, then these unused charges can be transformed into used ones, which supports the battery recovery effect, for recovering the remaining charge. The primary motivation behind this is to utilize the available resources of the battery up to its maximum limit and extend its lifetime, which must have sufficient capacity to hold the load. The main objectives of this paper are, first to enhance the battery lifetime of wearable devices in IoMT which increase the capacity of the battery and minimizes charge consumption during media transmission. Second, to utilize the recovery effect of the battery during discharging and to enhance the battery lifetime. Third, to test and deploy the proposed ABA and conventional battery recovery lifetime enhancement (BRLE) on the hardware.

The main aim of the paper is to empower the efficient and sustainable IoMT to fairly allocate the resources with the help of charge recovery, voltage scaling, and energy optimization up to the maximum level. In the IoMT the main part which consumes more energy is the communication, and due to dynamic features of the wireless channel more packet loss can be observed, and hence less reliability. Here, a novel IoMT framework and the hardware testbed of the proposed ABA are proposed.

This paper contributes remarkably in three distinct ways as follows.

- First, to propose a self-adaptive battery-aware algorithm based on the battery recovery effect principle in IoMT. The proposed ABA consumes less energy and enhances the battery lifetime up to $50 \mathrm{~s}$.

- Second, to propose a novel framework for IoMT based pervasive healthcare.

- Third, to test and deploy the proposed ABA and framework over hardware platform for energy efficient and longer battery lifetime in IoMT.

The remaining paper is structured as follows: Section 2 presents the detailed related works, the proposed data transmission framework is given in Section 3, and Section 4 discusses the adaptive battery-aware algorithm. The experimental results and discussion are revealed in Section 5, and finally paper is concluded in Section 6.

\section{Related Works}

The data transmission in emerging and intelligent healthcare plays the vital role for revealing clearer insight in the medical world. Additionally, rapidly evolving electrical grid technology plays a remarkable role in incorporating high penetration of renewable energy by establishing battery storage system. The modern adaptive state of charge (SoC) models the charge and discharge mechanism by avoiding the use of conventional methods, because these models are important to store more energy with high capacity. The smart 
grid controllers are based on the SoC forecast principle to manage and monitor the battery energy storage systems (BESS)'s operation. The three main BESS models are considered, two of them are reserved for parameters optimization and the third is reformulated for data content delivery and operation. The accuracies of both models are compared by properly selecting the required parameters $[4,5]$. The wearable sensors and IoT devices for healthcare are resource-constrained with limited capability to manage the resources, with less accuracy and reliability while monitoring and diagnosing the critical patients. As these tiny devices are used longer, the battery charge and power are increasingly drained. The electrochemical recovery effects of batteries consume more power from battery by applying idle-time in between occupied cycles. In practice, there is no evidence of how much the power is increased through the recovery effect, and it is not clear from most of the batteries and recent studies showed that the recovery effect does not exist at all. The experimental verification is presented for the clear battery recovery effect in wearable devices. The power management schemes that are used for the utilization of the recovery effect can increase the lifetime of the sensor devices $[6,7]$.

The batteries are non-linear in nature, however, to extend their lifetime is not equivalent to minimizing the consumption of energy $[8,9]$. The variation in battery features occur due to increase of the unused charges during the dissipation process. There are various battery charge optimizations and monitoring traits to extend the battery lifetime for effectively designing the future power-saving traits with the help of the voltage load. The analytical battery model for the battery recovery effect is a function of time. It is observed that the effect of idle time reduces battery charge depletion more and less for the proposed ABA and traditional BRLE accordingly. As energy saving and battery-driven methods are linearly related for the efficient, and enhanced scheduling and battery lifetime respectively in the IoMT, because lifetime extension of the entire network will impact the overall performance of the intelligent healthcare system. The battery features in association with the energy optimization methods give the accurate outcomes for examining the efficiency of both the hardware and software platforms. The adopted battery recovery principle in association with the proposed ABA enhances the IoMT lifetime with high energy efficiency and reliability, as the dynamic traits of the proposed ABA will lead to the adaptive battery behavior with time-invariant and linearly coordinated features. Furthermore, the battery response time is directly associating with its main recovery and discharging characteristics for enhancing the lifetime of IoMT system with more sustainability, reliability and high energy efficiency and less charge dissipation [10,11].

The battery behavior is analyzed with the help of proposed electrochemical driven recovery mechanism. The proposed model is stochastic and fundamental for achieving the adaptive mechanism with more deterministic battery monitoring mechanism. Overall, this can be possible through IoT-based wearable devices.

The key array-shaped electrochemical platform is considered with the support of some simple scheduling algorithms, where the discharge from each cell is accurately accumulated to optimize the recovery mechanism without initializing any extra delay. The battery management techniques give the improved performance of the system, so the parameter selection strategy will show less impact during battery cell behavior analysis [12].

The issue of energy-efficient packet scheduling in a wireless network is presented. The wireless transmitter is considered for managing the battery charge and capacity [13]. The battery lifetime is extended by introducing idle time, because it gives the optimal transmission schedule. This can be achieved by effective channel modeling and high energy efficiency by controlling the transmission power with longer duration [14,15]. In addition, the electrochemical behavior of the batteries will help the devices to recover more charge with extended lifetime. Two fundamental delay constraints, such as, deadline and aggregate driven for the battery-aware wearable devices.

Energy efficiency is an important challenge in intelligent wearable entities including Body Sensor Network (BSN) with lots of sensors on/around/implanted the patient's body [16]. The system-level consumption of the energy model is presented in coordination 
to the transmission distance and data contents over dynamic wireless channel [17]. The researchers in Nuggehalli et al. [18] present that a body area network (BAN) is used for systems of medical health care. The basic system for different applications which are established in BAN is a wireless sensor network (WSN) [19]. The emerging wireless technologies are empowering due to their new, practical, and innovative design with various sensors for proper monitoring of the healthcare applications and other medical systems [20-23]. The main challenge is to decrease the consumption of energy during the working of the node which is that the actual data transmission will be doing. The parameters duty cycle energy consumption for baseline, offline and recovery algorithm is calculated with the optimization algorithm as a genetic algorithm [24-27].

The main issue in mobile computing devices is high battery charge drain and shorter battery lifetime [28], as maximizing the lifetime of the battery is a critical issue because of the nonlinear behavior of the battery and its dependency on the discharging profile. Several issues were observed and examined while introducing voltage scaling, battery recovery, residual charge factors for obtaining the longer and sustainable IoMT [29]. The analytical battery model is adopted for recovering the battery charge and energy efficiency with optimized cost-function. The non-linear behavior of the battery consumes less charge, or saves more unused charge for enhancing the battery lifetime. To examine the battery features, charge and energy are optimized efficiently for the sustainable and recovered charge, hence extending the lifetime of IoMT [30].

We compared our proposed ABA with the traditional method named, battery recoverybased lifetime enhancement (BRLE) [31], which follows the battery voltage curve for monitoring and controlling the transcieving module of the nodes. Additionally, the transceiving module of the sensor node drains more energy in comparison to the other modules. The rechargeable batteries are used to power the wireless sensor node in near and remote hospitals and medical centers for proper diagnosing and examination of the patients, which is the challenging issue.

The battery charge recovery principle in association with the Markov model predicts the next battery charge level of cluster and child sensor nodes as cluster head $(\mathrm{CH})$ and cluster member (CM), further details are represented in Figure 2. The lifetime of the battery can be extended by properly following the time schedule of the transceiver module with potential entities, i.e., voltage curve of a battery, recovery factor, and distance between nodes. In addition, the conventional BRLE algorithm increases the throughput and avoids a connectivity loss or packet loss ratio in the network [31], because there is a limited battery capacity of mobile phones adopted in its functionality.

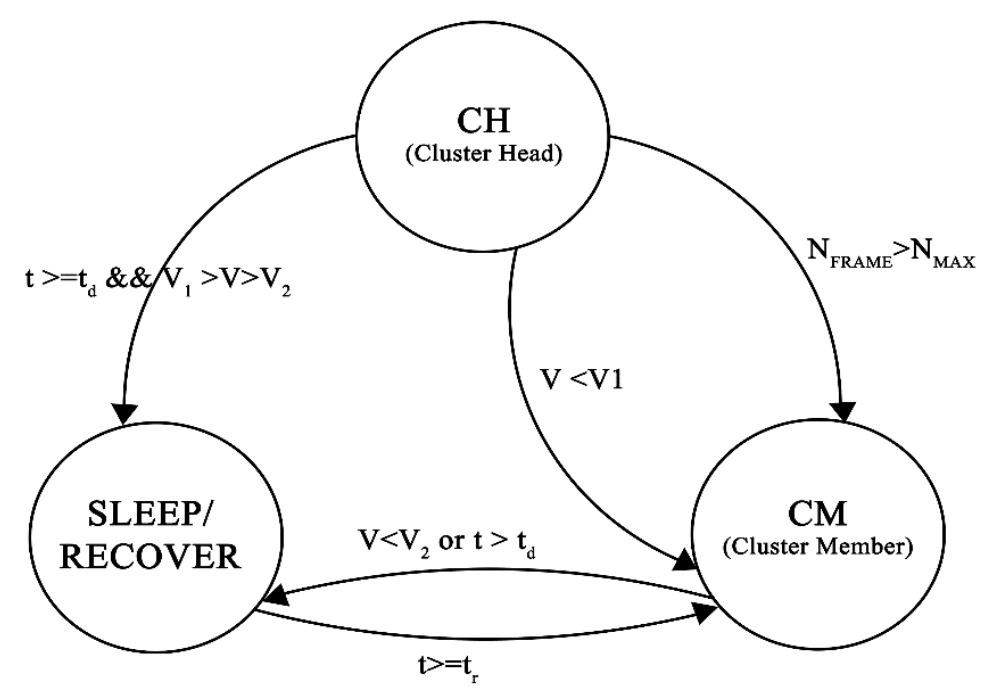

Figure 2. Battery recovery lifetime enhancement algorithm, The cluster head $(\mathrm{CH})$ and cluster member (CM) are key entities to recover the battery charge on the basis of voltage levels (V1, V2), time period $t$, duty-cycle $\left(t_{d}\right)$, and number of nodes/frame $\left(\mathrm{N}_{\text {frame }}, \mathrm{N}_{\max }\right)$ [31]. 
Moreover, the lifetime of the batteries reveals the longevity of a device, and the modeling of the battery can help to estimate and extend the lifetime. There are various battery models designed for the computing discharge rate of specific battery types and systems without involving workloads [31].

\section{Proposed Data Transmission Framework in IoMT Devices}

The continuous and sporadic nature of the medical data collected by the IoT-driven portable devices need more battery charge and hence, the energy is drained. The high image capacity and resource-constrained nature of the portable devices consume more battery charge while transferring data at remote location without any delay. For instance, if one physician wants to transfer the reports and medical images of one patient to another expert physician for consultation/getting advice at a remote location, it is vital to have efficient data delivery devices, as shown in Figure 2. In this way a logbook can be developed for the mentorship of the patients and future guidance of the other junior staff at hospitals and medical centers. In this way healthcare tips will be shared and exchanged to solve various issues of society.

The proposed medical data transmission are beneficial for patients at remote location, where resources are not accessible to the distant location villagers. Often, it is not easy and convenient for emergency patients to contact/reach the medical officers and hospitals for urgent treatment, consultation and necessary pre-cautions. The dramatic change and evolution of the digital world became the cornerstone of every field from academia to medical industries and hospitals. In the mean-time, sensor-driven wearable devices have facilitated the patients' lives globally by rectifying the traditional healthcare methods. The digitalization of the medical world not only gives comfort to the human lives by reducing the travel and treatment cost but also provides accurate disease detection and examination even in rural areas. Based on the highly recorded consultation lectures/advices, databases and medical reports, patients do not have to travel long distances, and they can easily get everything from highly qualified, skilled, and well-trained medical staff in their homes. The proposed intelligent data transmission platform is given detailed description as shown Figures 1 and 3, from both the software and hardware aspect accordingly. Both takes human vital sign signals such as ECG and medical images and gives output by efficiently allocating the resources at edge devices. There is a strong and reliable connectivity between the software and hardware testbeds for achieving an extended battery lifetime with a high reliability for the modern healthcare platform.

Many challenging medical issues are faced by patients in rural areas without any appropriate curable remedy. The state-of-the art portable devices for disease detection and patient's tracing are developed, but the key problem still faced by medical staff is the high charge drain and hence shorter battery lifetime of these wearable devices that lack proper monitoring of the battery status [29,30]. Multimedia transmission is the main entity to communicate between the hospitals and patients, clearly and effectively, anywhere with high visibility. In the case where the patient's condition is not critical, they will be consulted by an expert and well-trained medical staff through teleconferences for getting the necessary information and precautions. All the patients are diagnosed and treated by examining their vital sign signals, and these scans are forwarded to the required medical centers. The patient's symptoms scanning is a very important process for accurately visualizing the healthcare conditions, i.e., respiratory system, diabetic level, blood pressure and temperature. The sensing technologies have made the lives of parents comfortable because they can easily monitor their baby's health conditions by keeping records from respiration through to diaper change, which is possible through android applications in smart phones. This will consume more battery charge while transmitting the images and text contents. 


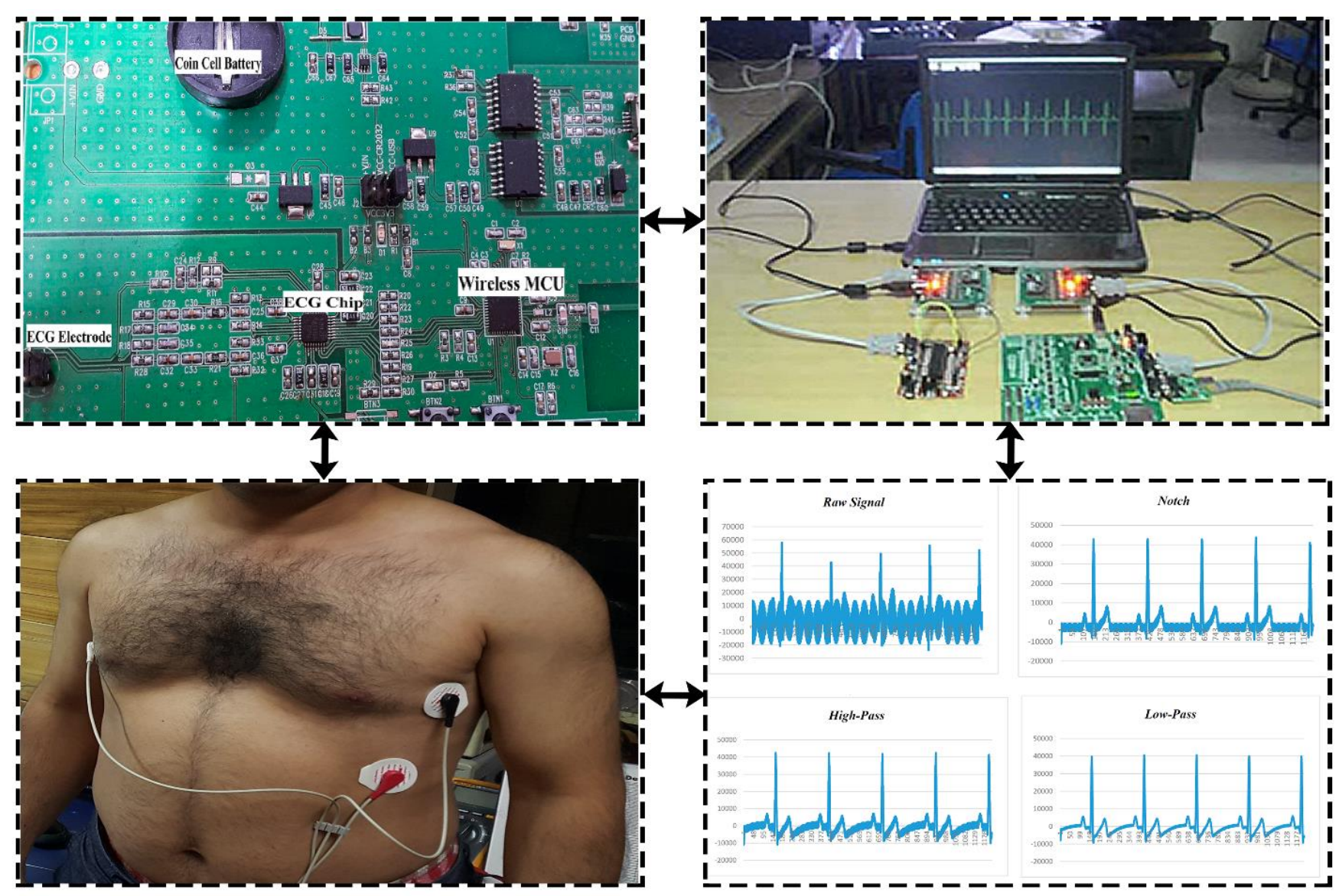

Figure 3. Hardware platform for human vital sign signal collection through the proposed battery-aware algorithm (ABA). Real-time ECG data samples are collected, filtered, analyzed, processed, and plotted. For further details see the detailed hardware implementation in our previous work in [2].

\section{Hardware Setup}

The hardware testbed is developed as shown in the Figure 3, by considering 30 subjects with no symptoms of heart-disease. The entire platform comprises ADS1292R circuit board (PCB) having CC2540F256 wireless micro control unit (MCU) and Bluetooth Low Energy (BLE) with $1 \mathrm{Mbps}$ physical data rate. Most of the experimental tests performed in the time schedule between morning and evening at $25^{\circ} \mathrm{C}$. The same developed hardware setup is used in our previous work [2]. The ECG collection and examination with detailed analysis of single-chip ECG signals is determined with the proper adaptation of filters. The key filter types for instance, notch, high pass, and low pass entirely rectify the collected ECG signals, and then compare the performance of the proposed ABA and traditional BRLE over both hardware and software setups. The hardware testbed is built by considering the patient's movement on a bicycle, i.e., the variation in the hear-rate. It is found that heart rate of the patients increases while driving the bicycle and gradually reduces as the speed slows. The performance of both the proposed ABA and the conventional BRLE is measured and examined in terms of the transmission power, received signal strength indicator (RSSI), battery lifetime, energy dissipation, charge optimization and so on. Additionally, Bluetooth low energy (BLE) dongle device wearable devices are used for collecting the ECG data while using the bicycle, so for validating the ECG data contents and removing the different noises from raw original contents, the ECG simulator with a power line noise, i.e., $50-60 \mathrm{~Hz}$ rectification is adopted. The developed ECG prototype adopts a high speed computer for gathering and transmitting relevant data through the BLE dongle device. Then the computer will exploit the rectified ECG signal for clear and accurate analysis, for further details readers can follow [2]. We consider the real-time datasets of the subjects collected through the BLE devices. Furthermore, adaptive high pass, low pass and notch filters are 
key role players in removing the unwanted noise. The proposed ABA optimizes the energy and battery charge level and extends the lifetime with the main recovery effect significantly unlike the traditional BRLE method. Because the proposed ABA creates the idle time for recovering the consumed charge, thus improving energy efficiency and battery lifetime.

\section{Proposed Adaptive Battery-Aware Algorithm}

The proposed adaptive battery aware algorithm (ABA) is based on the battery charge recovery principle for extending the IoMT lifetime, and hence the better and improved network performance. In this way the battery behavior and specific features are closely examined and evaluated to understand the working mechanisms of the entire healthcare system, as shown in Figure 3.

After initializing the battery parameters in Figure 4, it can be observed that when the voltage level goes below the upper threshold limit or threshold value (Vu equals to $3.2 \mathrm{~V}$ ), then the battery recovery charge and recovery time (i.e., idle-time) are calculated. In that idle time, the remaining unused charges will be recovered efficiently thus the voltage becomes higher than the $\mathrm{Vu}$, then the total charge dissipation will be computed. If the voltage level again gets below the lower threshold ( $\mathrm{Vl}$ as $2.2 \mathrm{~V}$ ), then several unused charges will be recovered then sleep mode will be adopted.

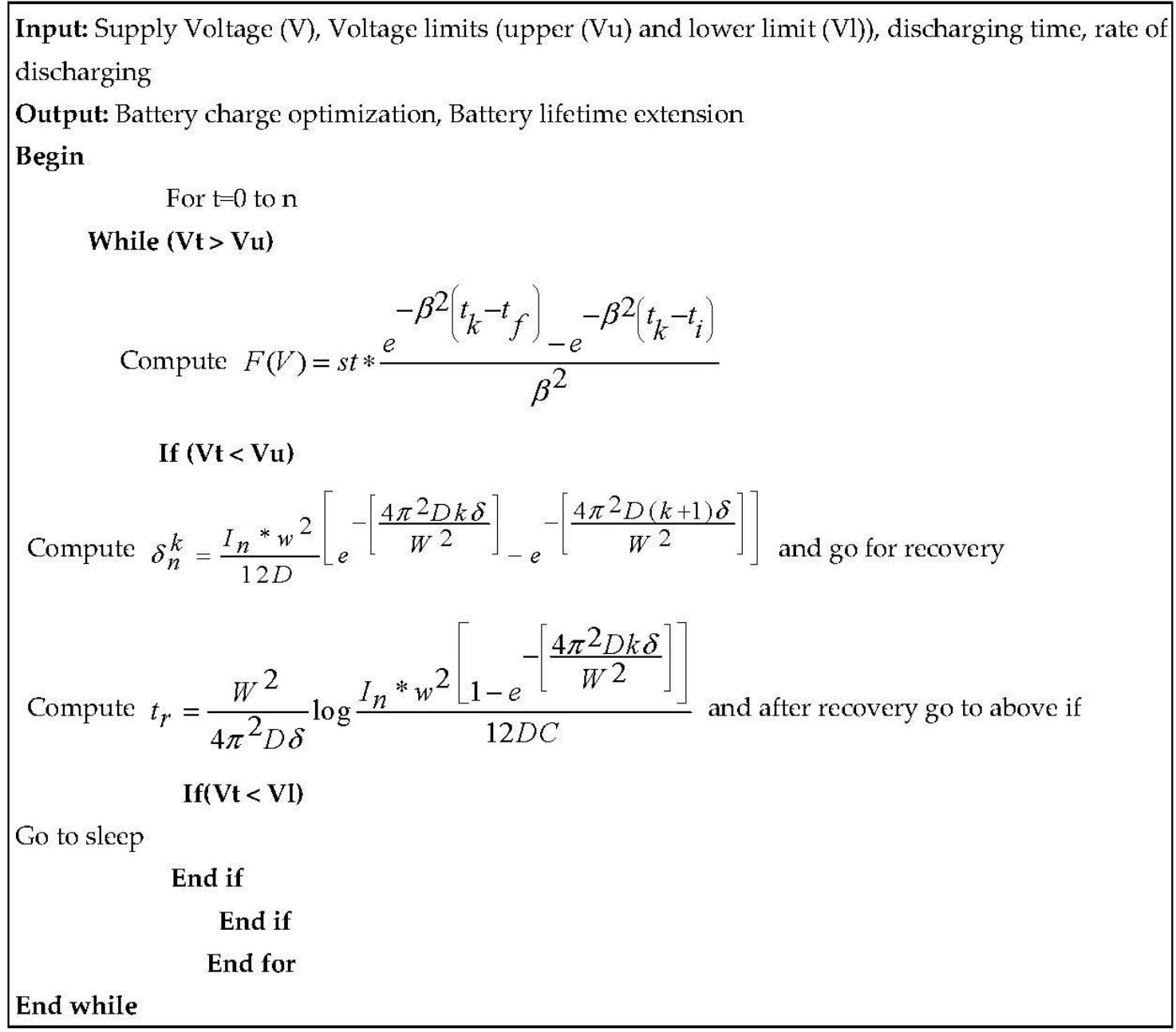

Figure 4. Proposed adaptive battery-aware algorithm, that optimizes the battery charge and its lifetime by considering the input parameters, i.e., voltage (threshold, upper and lower levels), batter discharge time and rate. 


\subsection{Analytical Battery Model}

The analytical battery model from Rakjmatov [11] is considered, which is based on diffusion principle and electrochemical reactions are computed as represented by Equation (1)

$$
\alpha=\int_{0}^{L} i(t) d t+\int_{0}^{L} i(t)\left(2 \sum_{m=1}^{+\infty} e^{-\beta^{2} m^{2}(L-t)}\right) d t
$$

Whereas $\alpha$ denotes the capacity of the battery, $\beta$ is the non-linearity of the battery, $i(t)$ gives the current profile and $L$ denotes the lifetime of the battery. For easy understanding and analysis, Equation (1) can be transformed into discrete form. Considering the load current arrangement into series of current values $I_{1}, I_{2} \ldots \ldots, I_{N}$ whereas, $I_{K}$ denotes the current for the $k$ th task at time $t_{k}$ with duration of time $\Delta k=t_{k+1}-1$. Furthermore, in order to better understand the characteristics of electrochemical batteries from Equation (1) the cost function $\sigma(t)$ over time $t$ is presented for defining the charge consumption process as shown in Equation (2)

$$
\sigma(t)=\sum_{k=1}^{M} I_{k} \Delta_{k}+\sum_{k=1}^{M} 2 I_{k} \sum_{m=1}^{\infty} \frac{e^{-\beta^{2} m^{2}}\left(e^{-\beta^{2} m^{2} \Delta_{k}-1}\right)}{\beta^{2} m^{2}} e^{-\beta^{2} m^{2} t_{k}}
$$

The charge consumption comprises two parts. The $l(t)$ linear part defines the total charge consumption which can be fully used by battery. The $u(t)$ non-linear part is nonnegative and defines the unavailable charges, i.e., some number of unused charges.

The discharging curve of the battery is modeled with the voltage function, which includes the state of charge (SOC) and exponentially decay process taken from Equation (2). The Equation (1) gives the mathematical expression of discharge curve when SOC equals st (in Equation (3)), and $S$ (in Equation (4)) is the remaining battery capacity.

$$
\begin{aligned}
& F(V)=s t * \frac{e^{-\beta^{2}\left(t_{k}-t_{f}\right)}-e^{-\beta^{2}\left(t_{k}-t_{i}\right)}}{\beta^{2}} \\
& F(V)=S * \frac{e^{-\beta^{2}\left(t_{k}-t_{f}\right)}-e^{-\beta^{2}\left(t_{k}-t_{i}\right)}}{\beta^{2}}
\end{aligned}
$$

Whereas, $F(V)$ is voltage function of the battery, $s$ denotes the decaying slop of SoC, $\mathrm{t}$ is the required battery discharging time, $\beta$ battery diffusion parameter, $t_{k}$ time required for $\mathrm{k}$ tasks, $t_{i}$ is the time for turning-on the load, $t_{f}$ time for turning-off the load. As the discharge process starts, the voltage of the battery decreases while getting below the threshold value, and in this situation, current drawn from battery will be decreased to adjust the load power. The discharging mechanism reduces the voltage level, which dissipates the entire energy amount quickly. The Equation (5) reveals the energy dissipation which includes the battery voltage and current.

$$
E=V_{b} * I_{b} * t_{d}
$$

Whereas, $V_{b}$ denotes the battery end voltage, $I_{b}$ is the current drained from battery and, $t_{d}$ is the discharge time to utilize the complete charges.

$$
E_{t o t}=\sum_{i=1}^{N}\left(E(b)+E_{t x_{i}}\left(b, d_{i j}\right)\right)
$$

Whereas, $E_{s e n_{i}}(b)$, and $E_{t x_{i}}\left(b, d_{i j}\right)$ are battery energy, and transmitting energy dissipation.

The discharge energy for the entire battery lifetime is the function of time and diffusion parameters, as represented by mathematical expression in Equation (7)

$$
F(T, n \delta,(n+1) \delta, \beta)=\delta+\frac{\pi^{2}}{3 \beta^{2}}\left[e^{-\beta^{2}(T-(n+1) \delta)}-e^{-\beta^{2}(T-n \delta)}\right]
$$


Whereas, $\delta$ is the slot length, and $\beta$ shows the diffusion parameter which is $\beta=\frac{2 \pi \sqrt{D}}{W}$ and it depends on the distance between the electrodes $(D)$ and width of the battery $(W)$. The charge dissipation will be computed when the load relates to the battery, and it determines the actual charge utilized amount, as given in Equation (8):

$$
F(T, n \delta,(n+1) \delta, \beta)=\delta+\frac{W^{2}}{12 D}\left[e^{-\left[\frac{4 \pi^{2} D}{W^{2}}\right](T-(n+1) \delta)}-e^{-\left[\frac{4 \pi^{2} D}{W^{2}}\right](T-n \delta)}\right]
$$

The battery recovery charge which relates the $I_{n}$ (current of $\mathrm{n}$ battery states), $w$ (depth of discharge), $W$ (width) and (D) distance between electrodes as revealed in Equation (9):

$$
\delta_{n}^{k}=\frac{I_{n} * w^{2}}{12 D}\left[e^{-\left[\frac{4 \pi^{2} D k \delta}{W^{2}}\right]}-e^{-\left[\frac{4 \pi^{2} D(k+1) \delta}{W^{2}}\right]}\right]
$$

Whereby, w is the depth of discharge (DOD) which means how much percentage of charges are remaining. The state of the charge and depth of discharge are inversely proportional. It can also be said that if SoC increases then DOD decreases and can be represented as $(D O D=100-S O C)$. The recovery length $t_{r}$ is modeled in Equation (10), where $C$ denotes the capacity of the battery.

$$
\begin{gathered}
t_{r}=\frac{W^{2}}{4 \pi^{2} D \delta} \log \frac{I_{n} * w^{2}\left[1-e^{-\left[\frac{4 \pi^{2} D k \delta}{W^{2}}\right]}\right]}{12 D C} \\
\text { DutyCycle }=\frac{T_{\text {tranON }}+T_{\text {Act }}+T_{\text {tranOFF }}}{T_{\text {tranON }}+T_{\text {Act }}+T_{\text {tranOFF }}+T_{\text {slp }}}
\end{gathered}
$$

$T_{\text {tranON }}$, time between sleep to idle mode, $T_{\text {Act }}$, time from Idle to sleep mode, $T_{\text {tranOFF }}$, active time of nodes, $T_{\text {slp }}$, sleep time of nodes. Then on the basis of these time schedules duty cycle is calculated in Equation (11). The Shannon capacity for transmission of packets is given by Equation (12):

$$
C=\frac{1}{2} \log _{2}\left(1+\frac{P}{N}\right) \text { bits } / \text { transmission }
$$

Whereas $P$ is average signal power constraint, and $N$ is noise power.

The number of transmissions required to transmit one bit is given by Equation (13):

$$
S=\frac{1}{C}
$$

From Equations (12) and (13), we will get Equation (14):

$$
P=s N\left(2^{\frac{2}{s}}-1\right)
$$

Assume that with single data byte transmission one joule of energy will be dissipated as shown in Equation (15):

$$
e(s)=s P=s N\left(2^{\frac{2}{s}}-1\right)
$$

Thus, energy depletion for transmitting one packet is shown in Equation (16):

$$
e(s)=\operatorname{LsN}\left(2^{\frac{2}{s}}-1\right)
$$

Whereas $L$ is the packet length in bits.

\subsection{Mealy Machines Based State Mechanism}

The mealy machines-based state mechanism is developed for changing states whose output values are determined both by its current state and the current inputs as shown in 
Figure 5. In the model the state of the sensor node is represented as $\mathrm{S}_{0}$ (Active element), while $S_{1}$ (battery element) and $S_{2}$ (sleep) are based on battery recovery process.

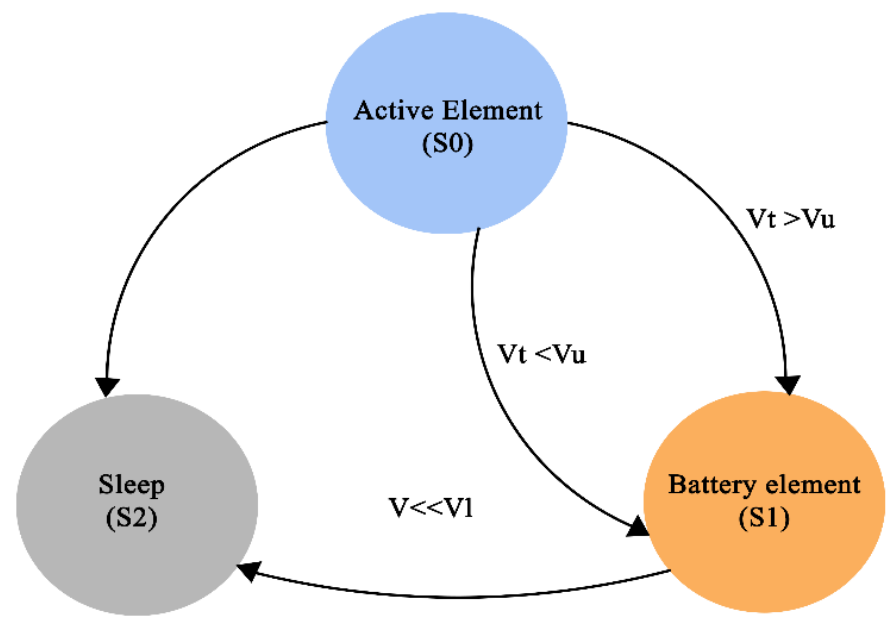

Figure 5. Mealy machine-based state transition mechanism, in this process state of the sensor node, i.e., $S_{0}$ (active element), $S_{1}$ (battery element), $S_{2}$ (sleep mode), and voltage levels (threshold and upper) are the main entities for battery recovery principal.

Figure 5 explains how the battery states are changing with the help of the adaptive mealy machines' mechanism. Initially the state will be in the active mode when the battery is fully charged and actively associated to the surface of electrodes. As the discharging process rapidly starts when the nearby electrodes are utilized at a lower voltage level, then battery state will be changed from the unused charge to the recovery charge with the insertion of some idle time. Thus, these battery elements reached the electrodes of the battery and utilizes the whole charge completely and then after that it goes to sleep. This recovery effect increases the lifetime of the battery by properly following the state changing mechanism, as illustrated in Figure 5.

\section{Results and Discussion}

This section presents the detailed experimental setup. The Convex optimization tool in MATLAB is adopted to implement both the proposed ABA and conventional methods, i.e., BRLE. Performance of the former and later is compared and analyzed in terms of networks metrics for instance, battery lifetime, energy dissipation, battery charge recovery, and lifetime of entire IoMT system, as revealed in Figures 6-10. Table 1 shows the detailed parameter list.

Table 1. Experimental entities and values.

\begin{tabular}{cc}
\hline Parameter & Value \\
\hline Battery used & Li-ion battery \\
Diffusion parameter $(\beta)$ & 0.31 \\
Time when load is on $t_{i}$ & 0 \\
Time when load is off $t_{f}$ & $3 \mathrm{~h}$ \\
Battery end voltage $V_{b}$ & $4.2 \mathrm{~V}$ \\
Current drawn from battery $I_{b}$ & $0.52 \mathrm{~A}$ \\
Distance between electrode $(D)$ & $18 \mathrm{~mm}$ \\
Width of the battery $(W)$ & $65.2 \mathrm{~mm}$ \\
Capacity of the battery & $2500 \mathrm{mAH}$ \\
Data & 32 bytes \\
Packet Length & 150 bytes \\
\hline
\end{tabular}




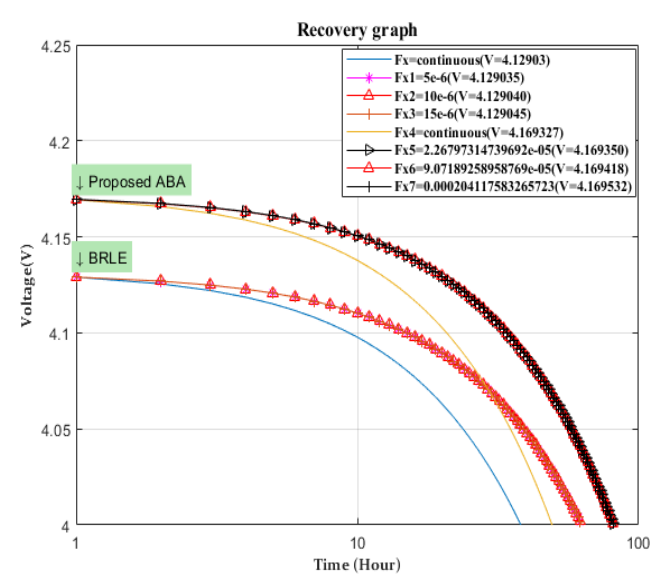

(a)

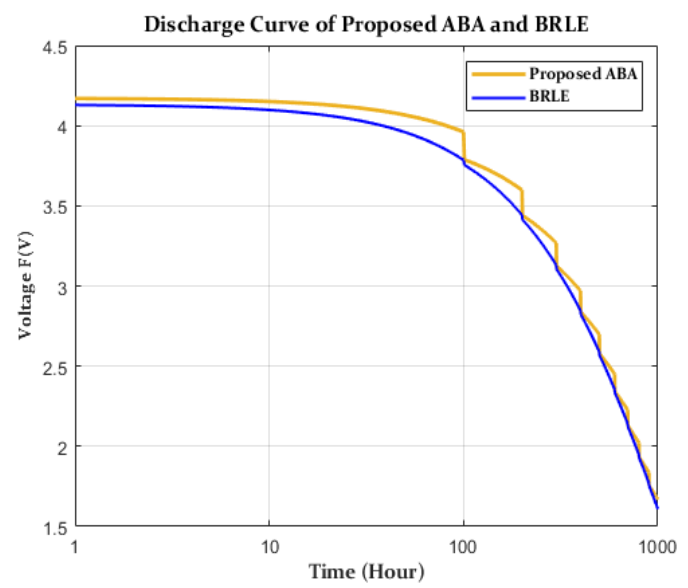

(b)

Figure 6. (a) Charge recovery, (b) discharge mechanism, tradeoff between time (hours) and voltage at different values is derived for both proposed ABA and conventional battery recovery lifetime enhancement (BRLE) to compute the charge recovery and discharge level.

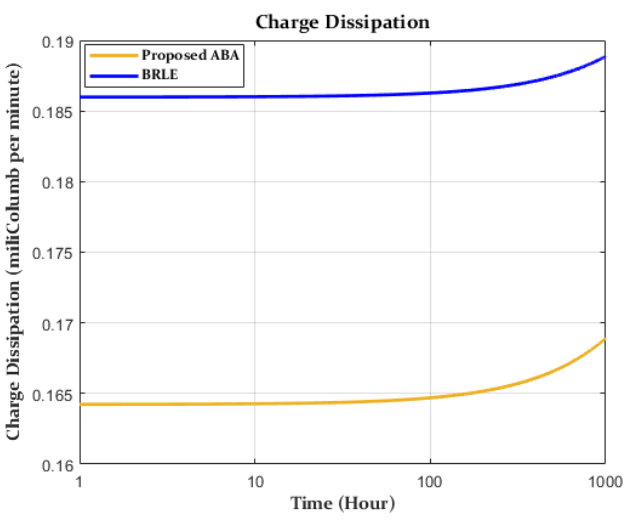

(a)

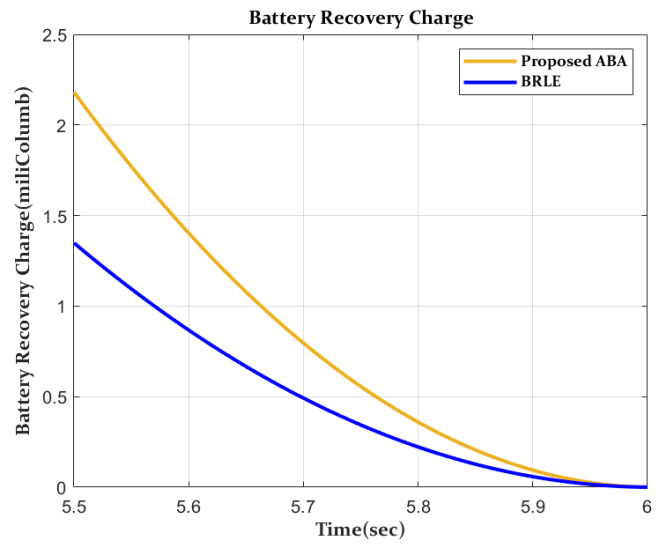

(b)

Figure 7. (a) Charge dissipation, (b) battery recovery charge, relationship between time and charge dissipation, and charge recovery are revealed for proposed ABA and Baseline BRLE.

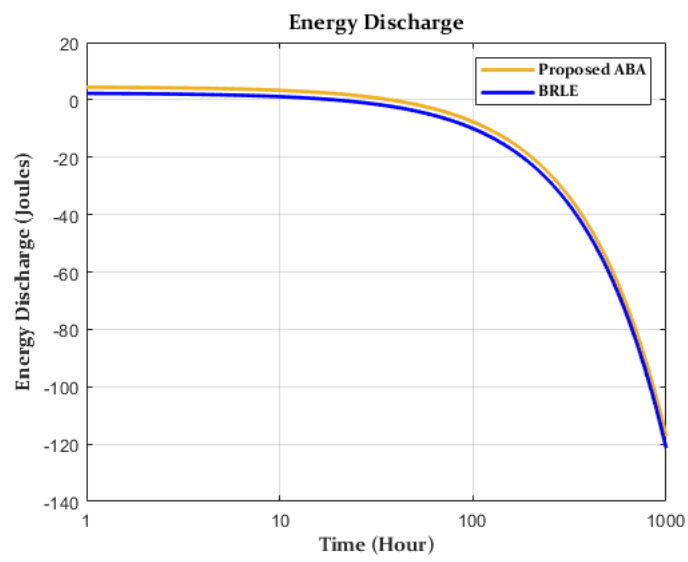

(a)

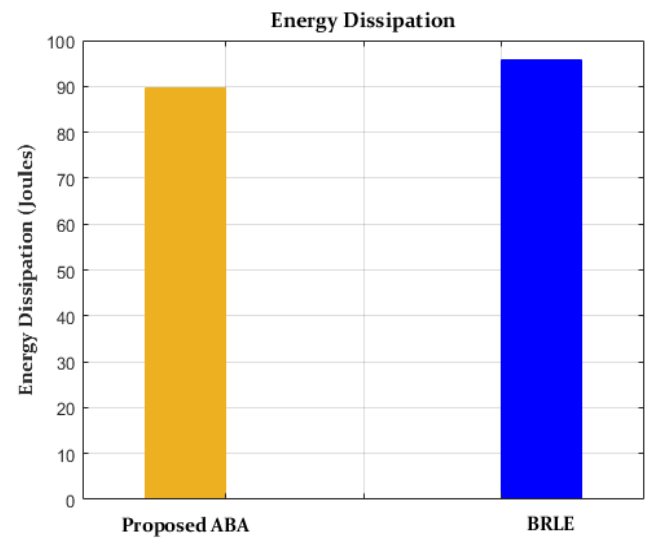

(b)

Figure 8. (a) Derives the relationship between time (hours) and energy discharge (joules) for the proposed ABA and BRLE. It is observed that the former has slightly less discharge than the later. (b) Reveals the energy dissipation pattern, where the former has less energy dissipation than the later. 


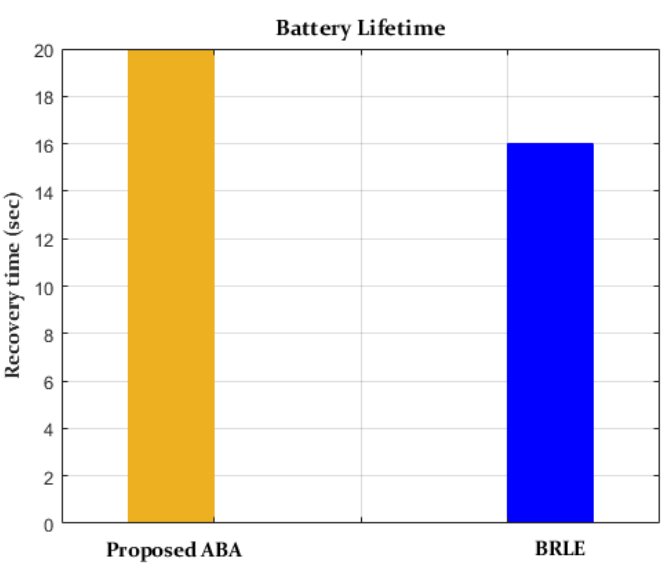

(a)

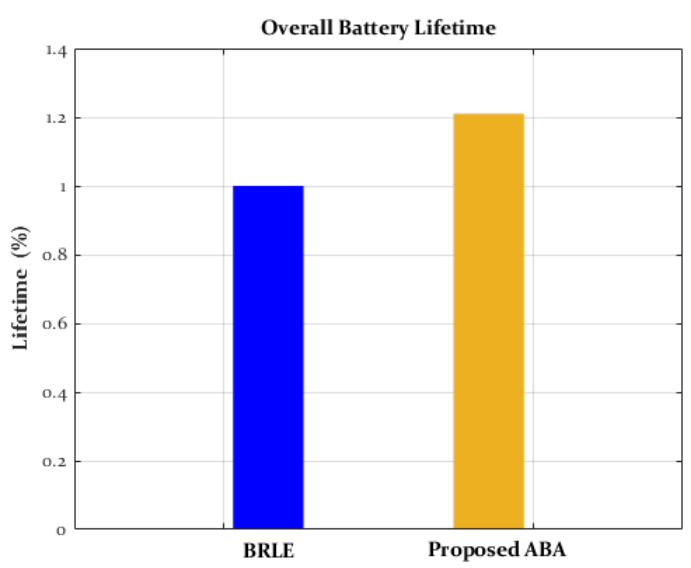

(b)

Figure 9. (a) Battery recovery time and (b) overall battery lifetime are revealed for the proposed ABA and BRLE. The analysis shows that the former has a longer recovery time and entire lifetime than the later.

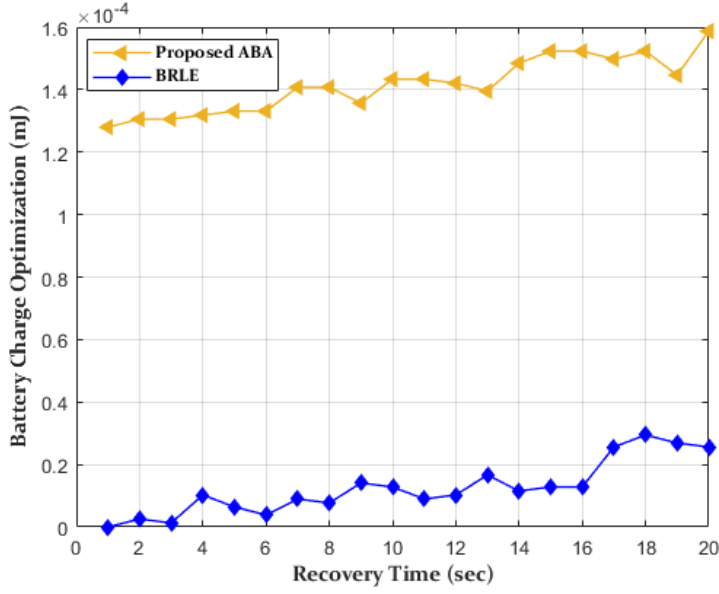

(a)

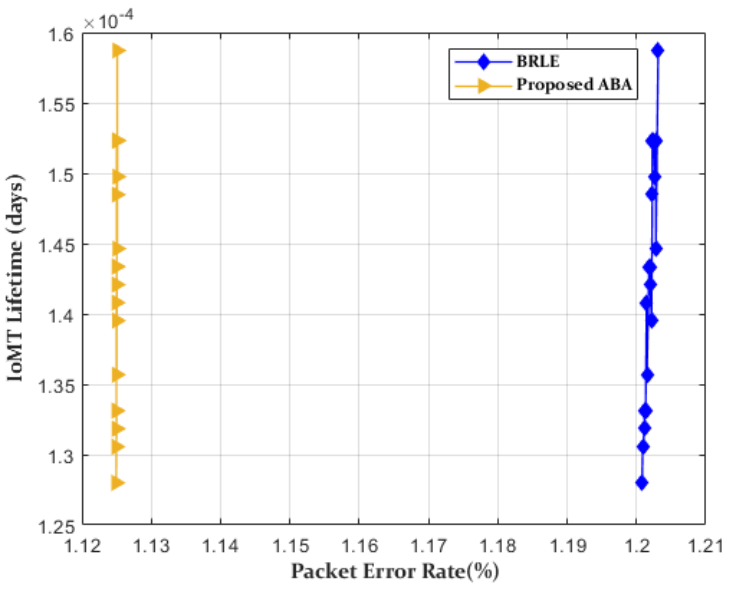

(b)

Figure 10. (a) The tradeoff between the recovery time (s) and battery charge optimization is revealed for the proposed ABA and BRLE. It is shown that the former optimizes the charge at a better level than the later. (b) The relationship between the packet error rate and battery lifetime in the IoMT is drawn. The lifetime shortens with the increase of the packet error rate, which is less for the former and higher for the later.

\section{Simulation Parameters}

The Figure $6 a, b$ shows the relationship between the time and voltage for the battery recovery, and discharge process, respectively. It is shown that high voltage is utilized in the battery recovery charge and discharge process sequentially. The entire statistics are extracted in Figure 6a and Table 2, with the linear coordination between the recovery charge and lifetime extension for IoMT.

Table 2. Comparison of recovery voltages.

\begin{tabular}{cccc}
\hline S. No & Battery Lifetime in (Seconds) & Starting Voltage for Recovery (Volts) & Voltage after Recovery (Volts) \\
\hline 1. & Continuous & 4.169327 & - \\
2. & 5 & 4.169327 & 4.169350 \\
3. & 10 & 4.169327 & 4.169418 \\
4. & 15 & 4.169327 & 4.169532 \\
5. & 20 & 4.169327 & 4.169532 \\
\hline
\end{tabular}


It can be observed that slightly more voltage is utilized by the proposed ABA than the conventional BRLE, as shown in Figure $6 \mathrm{~b}$. As we can see, there are voltage increases of up to 0.040297 for the proposed ABA algorithm. The battery charge recovery mechanism for the proposed ABA and traditional BRLE methods are presented in Figure 7a. It is shown that the former recovers more charge in a short period of time, unlike the later. The Figure $7 \mathrm{~b}$ shows the discharge curve of the proposed ABA and BRLE algorithms. Table 3 provides the data for the charge and energy dissipation. It is found that the charge dissipation of the proposed ABA is less than the traditional BRLE algorithm. The former recovers more battery charge, unlike the later. It is shown that with the increase of time span the recovery charges decreases, and after utilization of the entire charge it approximately becomes zero. Hence, we can say that maximum charges are utilized.

Table 3. Comparison of charge dissipation and energy dissipation.

\begin{tabular}{ccc}
\hline Algorithms & Charge Dissipation (mA.mint) & Energy Dissipation (J) \\
\hline Proposed ABA & $16,657.140903577998$ & 89.7 \\
BRLE & $18,742.6591179819989$ & 95.68 \\
\hline
\end{tabular}

Figure 8a shows the energy discharge in which the $x$-axis and $y$-axis show the time and energy discharge accordingly. The energy discharge of the batteries decreases with time. The battery powered devices could have a life of 3 to 4 years. After that the battery will not work properly and does not supply the charges to load. Figure $8 \mathrm{~b}$ shows that the proposed ABA algorithm consumes low energy, whereas the BRLE dissipates more energy. Hence, it can be said that former is a potential candidate for the IoMT, with a total recovery time of up to $50 \mathrm{~s}$, whereas the later recovers less charge with a shorter recovery time of $16 \mathrm{~s}$.

Figure $9 a, b$ shows the recovery time and battery lifetime, where the analysis shows that the proposed ABA has a high recovery time, while the traditional BRLE shows a shorter recovery time. Thus, there is less lifetime improvement of the later than the former. Therefore, it can be said that the proposed ABA extends the battery lifetime to a larger extent than the conventional BRLE, so the former is a promising potential candidate for the intelligent healthcare applications.

The Figure 10a presents the battery charge optimization in association with recovery time. It is observed that as the recovery time increases the remaining charge will be recovered significantly and more energy would be provided to the IoMT system. The calculated duty-cycle (87.9\%) gives battery capacity of $2500 \mathrm{mAh}$. The Figure 10b, shows the packet error rate for both proposed ABA and conventional BRLE algorithms. The packet error rate for the former is less, whereas for the later is higher, which means more data packets will be lost in the BRLE, unlike the proposed ABA. The packet error rate linearly increases with the number of cycles, at 1000 number of cycles the packet error rate will be increased to a large extent, and by reducing the number of cycles up to 10 , the packet error rate will be traduced as depicted in Figure 10b. Hence, it can be said that the packet error rate is linearly proportional to the battery lifetime extension of the IoMT system. The comparison of previous techniques is given in Table 4 .

The sporadic and unscheduled data interruption during transmission and communication creates unplanned outages, and it is mostly observed in the main transmission part of the IoMT. Due to the higher energy depletion in the nodes with proximity to the sink region, these nodes will die sooner from outer sub-region, because they send their own data and also forward outer sub-regions data to the sink. This will create the energy hole near to the sink region due to the shorter time, thus, the proposed ABA algorithm increases the throughput and reduces the unplanned outages and hotspot. 
Table 4. Comparison of previous techniques for the Internet of medical Things (IoMT).

\begin{tabular}{|c|c|c|c|}
\hline References & $\begin{array}{l}\text { Existing and Proposed } \\
\text { Algorithms }\end{array}$ & Strengths & Weaknesses \\
\hline [15] & $\begin{array}{l}\text { A Bi-Level Optimization } \\
\text { Approach to Charging Load } \\
\text { Regulation of Electric Vehicle } \\
\text { Fast Charging Stations Based on } \\
\text { a Battery Energy Storage System }\end{array}$ & $\begin{array}{l}\text { Battery energy storage system } \\
\text { (BESS) for charging load control, } \\
\text { which is a more user-friendly and } \\
\text { robust approach to perturbations }\end{array}$ & $\begin{array}{l}\text { Does not focus on battery } \\
\text { charge recovery, lifetime } \\
\text { extension and reliability } \\
\text { in healthcare }\end{array}$ \\
\hline [25] & $\begin{array}{c}\text { Hierarchical Energy } \\
\text { Optimization Strategy and its } \\
\text { Integrated Reliable Battery Fault } \\
\text { Management for Hybrid } \\
\text { Hydraulic-Electric Vehicle }\end{array}$ & $\begin{array}{l}\text { Suboptimal control strategy based } \\
\text { on fuzzy logic and neural } \\
\text { network for minimizing total } \\
\text { energy consumption while } \\
\text { ensuring a better battery life }\end{array}$ & $\begin{array}{l}\text { Emergency healthcare, and } \\
\text { reliability with hardware } \\
\text { platform are not discussed. }\end{array}$ \\
\hline [31] & $\begin{array}{l}\text { Battery Recovery Based } \\
\text { Lifetime Enhancement (BRLE) } \\
\text { Algorithm for Wireless } \\
\text { Sensor Network }\end{array}$ & $\begin{array}{l}\text { Transceiver module based on the } \\
\text { battery terminal voltage, recovery } \\
\text { factor and distance between the } \\
\text { nodes, the lifetime of the network } \\
\text { is enhancing }\end{array}$ & $\begin{array}{l}\text { Does not consider discharge } \\
\text { time and recovery time of the } \\
\text { battery in a } \\
\text { real-time environment. }\end{array}$ \\
\hline [16] & $\begin{array}{l}\text { An energy efficient wireless } \\
\text { body area network using } \\
\text { genetic algorithm. }\end{array}$ & $\begin{array}{l}\text { Genetic algorithm used to } \\
\text { optimize the energy consumption } \\
\text { in WBAN }\end{array}$ & $\begin{array}{c}\text { Battery charge recovery, and } \\
\text { hardware setup are } \\
\text { not considered. }\end{array}$ \\
\hline [27] & $\begin{array}{l}\text { An energy-efficient fog-to-cloud } \\
\text { Internet of Medical } \\
\text { Things architecture }\end{array}$ & $\begin{array}{l}\text { Bluetooth technology-based } \\
\text { energy efficient sleep and } \\
\text { wake-up modes }\end{array}$ & $\begin{array}{c}\text { Does not focus on battery } \\
\text { charge recovery, reliability and } \\
\text { hardware setup }\end{array}$ \\
\hline$[1,21]$ & $\begin{array}{l}\text { A Multi-sensor Data Fusion } \\
\text { Enabled Ensemble Approach for } \\
\text { Medical Data from Body } \\
\text { Sensor Networks }\end{array}$ & $\begin{array}{l}\text { QoS optimization in terms of } \\
\text { mobility, reliability, and packet } \\
\text { loss ratio for BSNs }\end{array}$ & $\begin{array}{l}\text { Does not consider the battery } \\
\text { recovery and discharging time, } \\
\text { and hardware test-bed }\end{array}$ \\
\hline Our Proposed ABA & $\begin{array}{l}\text { A Novel Adaptive } \\
\text { Battery-Aware Algorithm for } \\
\text { Data Transmission in IoT-based } \\
\text { Healthcare Applications }\end{array}$ & $\begin{array}{l}\text { The proposed ABA implements } \\
\text { and adopts the recovery effect for } \\
\text { enhancing energy efficiency, } \\
\text { battery lifetime, reliability, } \\
\text { throughput and reduces the } \\
\text { unplanned outages and hotspot }\end{array}$ & $\begin{array}{l}\text { Slightly more complexity in } \\
\text { hardware setup and more } \\
\text { processing time }\end{array}$ \\
\hline
\end{tabular}

\section{Conclusions and Future Work}

The battery's properties of lifetime extension, energy optimization and high reliability are the key challenges in the IoMT based intelligent healthcare in today's era. Thus, this research contributes three main contributions, first, a novel adaptive battery-aware algorithm (ABA) is proposed, which utilizes the charges up to its maximum limit and recovers those which are unused. The proposed ABA adopts this recovery effect for enhancing energy efficiency, battery lifetime, throughput, and reliability. Second, to propose a novel framework for the IoMT based intelligent pervasive healthcare applications. Third, to test and deploy the proposed ABA and framework over a hardware platform and compare it with the state-of-the art conventional BRLE method for comparing energy efficiency and battery lifetime extension in the IoMT. The proposed ABA algorithm increases the lifetime of the battery and provides high performance as compared to the conventional BRLE algorithm, and the former consumes low energy and supports the continuous connectivity of devices, unlike the later. The analytical model of the Li-ion battery is considered, and an extensive experimental setup is performed which shows that the proposed ABA algorithm outperforms the BRLE by reducing the energy drain. The proposed ABA recovers $50 \%$ more charge and hence has a longer battery lifetime than the traditional BRLE (0.0598). The extracted experimental results reveal that more charge is recovered, and hence there is a longer battery lifetime with high reliability, with a smaller packet error rate, with enhanced energy efficiency by the proposed ABA as compared to the traditional BRLE 
method. Due to the usage of the battery, pushed to its maximum limit in the proposed $\mathrm{ABA}$, it requires protection to handle the high voltages and it has long sleep durations for achieving the high charging time. Hence, it can be said that our proposal is a very promising and potential candidate for the pervasive and intelligent healthcare application in the IoMT system.

In the near future we will adopt the proposed ABA for real-time multimedia applications in the hospitals at remote locations over 5G-driven wearable devices.

Author Contributions: H.M., A.H.S., and M.S.A.-R. worked on technical writing, Introduction section and supervision of the entire technical research theme respectively. N.Z. prepared the literature review; S.P. and L.W. performed the experiment, compiled the results, and composed the manuscript with great supervision the field activities and designed the study's analytic strategy. All authors have read and agreed to the published version of the manuscript.

Funding: This work is financially supported by the Deanship of Scientific Research, Research Chair of Pervasive and Mobile Computing, King Saud University, Riyadh, Saudi Arabia, and by Research grant of PIFI 2020 (2020VBC0002), China.

Conflicts of Interest: The authors declare no conflict of interest.

\section{References}

1. Muzammal, M.; Talat, R.; Pirbhulal, S. A Multi-sensor data fusion enabled ensemble approach for medical data from body sensor networks. Inf. Fusion 2020, 53, 155-164. [CrossRef]

2. Sodhro, A.H.; Pirbhulal, S.; Sanagiah, A.K. An energy-efficient algorithm for wearable electrocardiogram signal processing in ubiquitous 3 healthcare applications. Sensors 2018, 8, 923. [CrossRef] [PubMed]

3. Magsi, H.; Sodhro, A.H.; Chachar, F.A.; Khan Abro, S.A.; Sodhro, G.H.; Pirbhulal, S. Evolution of 5G in Internet of medical things. In Proceedings of the 2018 International Conference on Computing, Mathematics and Engineering Technologies (iCoMET), Sukkur, Pakistan, 3-4 March 2018.

4. Rosewater, D.; Ferreira, S.; Schoenwald, D.; Hawkins, J.; Santoso, S. Battery Energy Storage State-of-Charge Forecasting: Models, Optimization, and Accuracy. In IEEE Transactions on Smart Grid; IEEE: Piscatavai, NJ, USA, 2018; pp. $2453-2462$.

5. Hume, R.; Looney, J. Telemedicine and facility design. Health Facil. Manag. 2016, 29, 30.

6. Motaqi, A.; Mosavi, M.R. Blind and task-ware multi-cell battery management system. Eng. Sci. Technol. Int. J. 2019, 23, 544-554. [CrossRef]

7. Lin, C.W.; Pan, C.J.; Paranjape, K.; Yu, C.; Feng, P. Taipei Citizen Telecare Service System for Hypertension Management in Elders. In Proceedings of the 2014 Annual SRII Global Conference, Washington, DC, USA, 23 April 2014.

8. Sodhro, A.H.; Obaidat, M.S. Quality of service optimization in IOT driven intelligent transportation system. IEEE Wirel. Commun. Mag. 2019, 26, 10-17. [CrossRef]

9. Jongerden, M.R.; Haverkort, B.R. Battery Modeling. 2008. Available online: https://www.researchgate.net/publication/239851 603_Battery_Modeling (accessed on 3 February 2021).

10. Rakhmatov, D.; Vrudhula, S.; Wallach, D.A. A model for battery lifetime analysis for organizing applications on a pocket computer. IEEE Trans. Very Large Scale Integr. VLSI Syst. 2003, 11, 1019-1030. [CrossRef]

11. Rao, R.; Vrudhula, S. Battery optimization vs energy optimization: Which to choose and when? In Proceedings of the 2005 IEEE/ACM International conference on Computer-Aided Design, San Jose, CA, USA, 6-10 November 2005.

12. Sodhro, A.H.; Li, Y.; Shah, M.A. Green and friendly media transmission algorithms for wireless body sensor networks. Multimed. Tools Appl. 2017, 76, 20001-20025. [CrossRef]

13. Sodhro, A.H.; Li, Y.; Shah, M.A. Energy-efficient adaptive transmission power control for wireless body area networks. IET Commun. 2016, 10, 81-90. [CrossRef]

14. Bao, Y.; Luo, Y.; Zhang, W.; Huang, M.; Wang, L.Y.; Jiang, J. A bi-level optimization approach to charging load regulation of electric vehicle fast charging stations based on a battery energy storage system. Energies 2018, 11, 229. [CrossRef]

15. Kaur, H. An Energy efficient wireless body area network using Genetic algorithm. Int. J. Adv. Res. Comput. Sci. 2017, 8, 98-106. [CrossRef]

16. Chiasserini, C.-F.; Ramesh, R.R. Energy efficient battery management. IEEE J. Sel. Areas Commun. 2001, 19, 1235-1245. [CrossRef]

17. Sufian, H.; Khan, F.I.; Hameed, B. Understanding security requirements and challenges in internet of things (IoT): A review. J. Comput. Netw. Commun. 2019, 2019. [CrossRef]

18. Nuggehalli, P.; Vikram, S.; Ramesh, R.R. Energy efficient transmission scheduling for delay constrained wireless networks. IEEE Trans. Wirel. Commun. 2006, 5, 531-539. [CrossRef]

19. Yi, C.; Wang, L.; Li, Y. Energy efficient transmission approach for WBAN based on threshold distance. IEEE Sens. J. 2015, 15, 5133-5141. [CrossRef] 
20. Sodhro, A.H.; Mohsen, G.; Boukerche, A. AI-enabled reliable channel modelling architecture for fog computing vehicular networks. IEEE Wirel. Commun. Mag. 2020, 27, 14-21. [CrossRef]

21. Dai, Y.; Wang, G.; Muhammad, K.; Liu, S. A closed-loop healthcare processing approach based on deep reinforcement learning. Multimed. Tools Appl. 2020,1-23.

22. Fourati, L.C. Wireless Body Area Network and Healthcare Monitoring System. In Proceedings of the 2014 IEEE International Conference on Healthcare Informatics, Verona, Italy, 15-17 September 2014.

23. Ramli, S.N.; Rabiah, A. Surveying the wireless body area network in the realm of wireless communication. In Proceedings of the 7th International Conference on Information Assurance and Security (IAS), Melaka, Malaysia, 5-8 December 2011.

24. Kamal, E.; Adouane, L. Hierarchical energy optimization strategy and its integrated reliable battery fault management for hybrid hydraulic-electric vehicle. IEEE Trans. Veh. Technol. 2018, 67, 3740-3754. [CrossRef]

25. Sodhro, A.H.; Pirbhulal, S. Artificial intelligence driven mechanism for edge computing based industrial applications. IEEE Trans. Ind. Inform. 2019, 15, 4235-4243. [CrossRef]

26. Tahir, S.; Bakhsh, S.T.; Abulkhair, M.; Alassafi, M.O. An energy-efficient fog-to-cloud internet of medical things architecture. Int. J. Distrib. Sens. Netw. 2019, 15. [CrossRef]

27. Liu, X.; Lin, C.C.; Muhammad, K.; Al-Turjman, F.; Yuan, S. Joint data hiding and compression scheme based on modified BTC and image inpainting. IEEE Access 2019, 7, 116027-116037. [CrossRef]

28. Darwish, A.; Hassanien, A.E.; Elhoseny, M.; Sangaiah, A.K.; Muhammad, K. The impact of the hybrid platform of internet of things and cloud computing on healthcare systems: Opportunities, challenges, and open problems. J. Ambient Intell. Humaniz. Comput. 2019, 10, 4151-4166. [CrossRef]

29. Tianle, Z.; Sodhro, A.H.; Luo, Y. A joint deep learning and internet of medical things driven framework for elderly patients. IEEE Access 2020, 8, 75822-75832.

30. Shabir, A.P.; Javaid, A.S. Secure data transmission framework for confidentiality in IoTs. Ad Hoc Netw. 2019, 95, 101989.

31. Mahima, V.; Chitra, A. Battery recovery based lifetime enhancement (BRLE) algorithm for wireless sensor network. Wirel. Pers. Commun. 2017, 97, 6541-6557. [CrossRef] 